\title{
Automatic Vertebrae Localization and Spine Centerline Extraction in Radiographs of Patients with Adolescent Idiopathic Scoliosis
}

\author{
Gunarajulu RENGANATHAN ${ }^{\mathrm{a}}$, Navin MANASWI ${ }^{\mathrm{b}}$, Ionuţ GHIONEA ${ }^{\mathrm{c}}$ and \\ Sasa CUKOVIC ${ }^{\mathrm{d}, 1}$ \\ anna University, Chennai, India \\ ${ }^{\mathrm{b}}$ WOWExp Technologies, Bangalore, India \\ ${ }^{\mathrm{c}}$ University Politehnica of Bucharest, Faculty of Industrial Engineering and Robotics, \\ Romania \\ ${ }^{\mathrm{d}}$ Swiss Federal Institute of Technology - ETH Zurich, Institute for Biomechanics, \\ Switzerland
}

\begin{abstract}
Adolescent Idiopathic Scoliosis (AIS) is lifetime disorder indicated by the abnormal spinal curvature, and it is usually detected in children and adolescents. Traditional radiographic assessment of scoliosis is time-consuming and unreliable due to high variability in images and manual interpretation. Vertebrae localization and centerline extraction from a biplanar X-ray is essential for pathological diagnosis, treatment planning, and decision making. The aim of this paper is to develop a fully automated framework to provide correct evaluation of anatomical landmarks and to extract vertebral and intervertebral discs' centroids. By knowing coordinates of each centroid, developed framework will estimate 2D deformity curve (centerline) called Middle Spinal Alignment (MSA) in frontal plane. By analyzing the MSA lines and deformity segments, many deformity parameters can be calculated which include vertebral transpositions, Cobb angles, apex vertebra position, etc., for planning spinal correction strategies and monitoring.
\end{abstract}

Keywords. Adolescent Idiopathic Scoliosis, Landmark Detection, Centroids Localization, Middle Spinal Alignment

\section{Introduction}

Adolescent Idiopathic Scoliosis (AIS) is a structurally deviated, lateral and axially rotated deformity of the spine, a lifetime condition that usually arises in children or around puberty [1]. Conventional early detection, physical therapy and bracing would be preferred to increase the quality of life, reduce deformation and to prevent surgery.

The early diagnosis would be an appropriate choice that limits the disorder progression and provides positive impacts on prognosis and treatment. It is traditionally evaluated using the Anterior-Posterior (AP) or biplanar radiographic (X-ray) images [2].

\footnotetext{
${ }^{1}$ Corresponding Author, Sasa Cukovic, Swiss Federal Institute of Technology - ETH Zurich, Institute for Biomechanics, Leopold-Ruzicka Weg 4, 8093 Zurich, Switzerland; E-mail: sasa.cukovic@hest.ethz.ch.
} 
Although traditional measurement involves clinical intervention in identifying the vertebrae structures, high time-consuming, high inter- and intra- observer variability measurements are usually affected by vertebrae selection, observer bias and image quality. To increase precision of investigating scoliosis over X-ray radiographs, some image processing approaches were developed and they could be generally classified as: 1) unsupervised and 2) supervised.

\subsection{Unsupervised approaches for AIS Evaluation}

The most promising computer-aided methods proposed in the literature for the scoliosis line estimation are Active Contour (AC) and filtering. These approaches are appropriate to localize the end vertebrae of the deformity segments and to calculate Cobb angles and thus to describe curvature. However, these methods are parameter sensitive and computationally expensive.

\subsection{Supervised approaches for AIS Evaluation}

To overcome abovementioned limitations, supervised approaches such as Support Vector Regression (SVR), Random Forest Regression (RFR), and Convolutional Neural Networks (CNN) were developed. The main disadvantage of these approaches are limited field of view (FOV) and additional preprocessing strategies in case of the metal implants presence on X-ray images. Nevertheless, these approaches performed well with annotated features [3]. To address these specific challenges using CNN, convolutional layers were employed to segment the vertebrae, but still the image sensitivity and quality play a vital role.

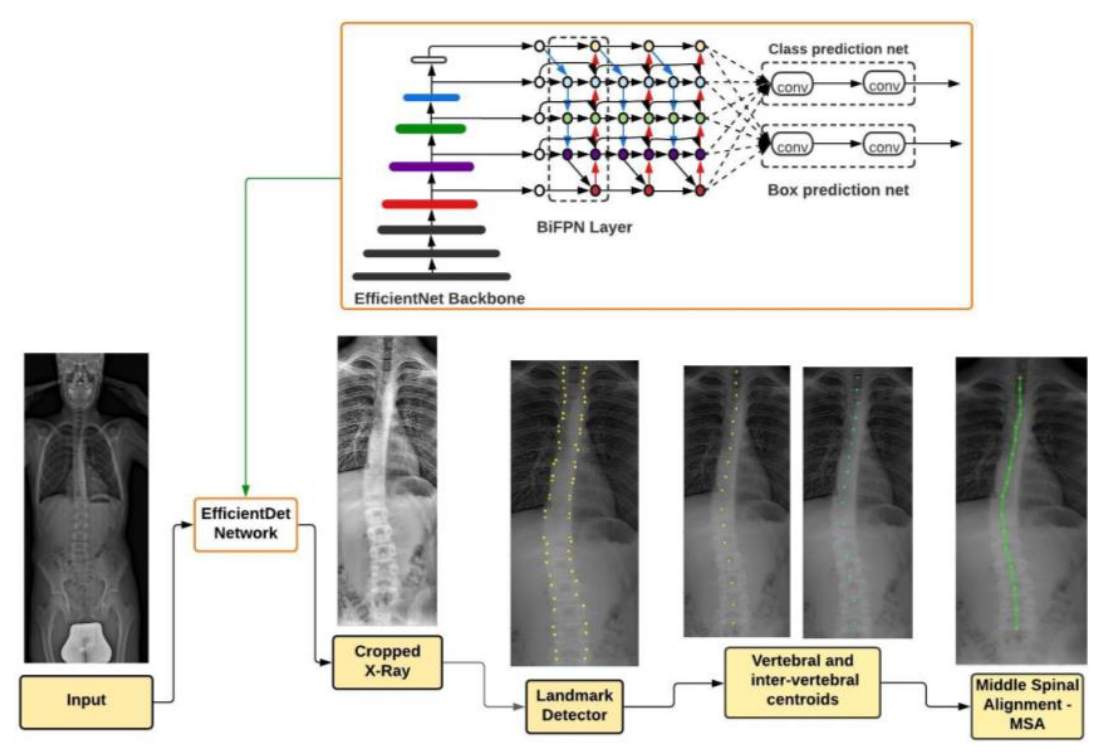

Figure 1. Proposed framework for the vertebral landmarks localization and MSA extraction based on a spinal focused (cropped) image

Advanced object detection techniques like EfficientDet have been used as an initial stage to localize vertebrae. The most promising object detection model called Faster - 
RCNN (Region-based Convolutional Neural Network) to estimate vertebral landmarks and MSA for scoliosis assessment including centroid coordinates was implemented [2]. Figure 1 illustrates our proposed framework.

As a result of image processing and estimating vertebral anatomical points using landmark-detection framework, we were able to generate coordinates of vertebral and intervertebral discs' centroids in AP plane and to evaluate MSA line as a spinal centerline or spinal alignment.

The main advantage of the proposed landmark detection network is that it is more accurate compared to other methods. The main disadvantages are that the input images need to be cropped and focused on the spinal regions and that the image size variants may create false positive results in localization.

\section{Materials and Methods}

Our framework was trained for X-ray images that focus only the spinal area (i.e., spinal structures are isolated from the original images). Initially, we manually cropped the unwanted body parts from all X-ray images so that they can match the input criteria to the landmark-detector network. In next stage, we improved the framework with a preprocessing network that act as a filter to eliminate the other anatomical regions (i.e., skull and lower limb) and to properly detect the landmarks with less time consumed.

The latest deep learning network called EfficientDet Network was recently proved to be one of the best for object detection tasks as it produces a high accuracy feature detection within less time [4]. The selected network also has many variants (EfficientDetD0 to D6) based on accuracy and inference time out of which, D0 variant is used due to lack of high-end PC configuration to train the network. This design proposed a weighted bidirectional feature network for scaling, which also optimizes the features for better accuracy and efficiency. Then the object detector EfficientDet model was used to detect the vertebrae as bounding box objects which were then given to the landmark detector network separately [5]. Hence to estimate the MSA for vertebral centroid estimation, four landmark corner points were taken as input, through which, intervertebral centroids are calculated. Predicted landmark coordinates are mapped to the original images to estimate the frontal scoliosis profile.

\subsection{Datasets}

We use training data (480 images) from the SpineWeb [2] as our training dataset. The testing images (100 images) were given by Clinical center Kragujevac (Serbia) from previous studies [7]. All testing and training images are the AP X-ray radiographs. As a primary inclusion criterion, we considered subjects with adolescent idiopathic scoliosis (AIS) with proper X-ray images from the first clinical assessment. Exclusion criteria were subjects with spinal surgery and vertebral implants, congenital scoliosis, adults, pregnant women and images with low intensity/quality (16 images of such criteria are eliminated).

In specific, we use $60 \%$ (348 images), $20 \%$ for validation (116 images) and $20 \%$ for testing (100 images). Each image was detected with 17 vertebrae from the thoracic and lumbar spine and the 4 landmark corners were localized in each vertebra (68 points per image). The input image varies in resolution around $\sim 2000 \times 4500$ pixels. 


\section{Results and Discussion}

The segmentation-based algorithms failed to address the separation of fused (congenitally connected) vertebrae and the false prediction rates were high in that case. For our clinical datasets, the results were quite reliable even in a low contrast images because the vertebrae focused landmarks and outlier rejections were carried out in the preprocessing stage. It also helped the object detection network to map corner points of each vertebral body and thus increased the accuracy rate.

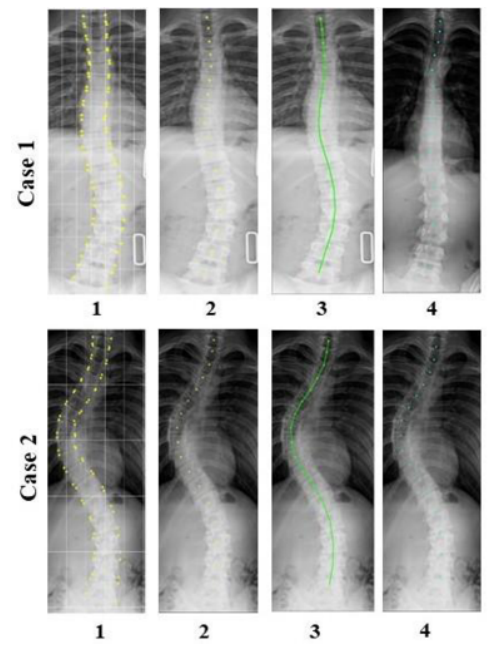

a)

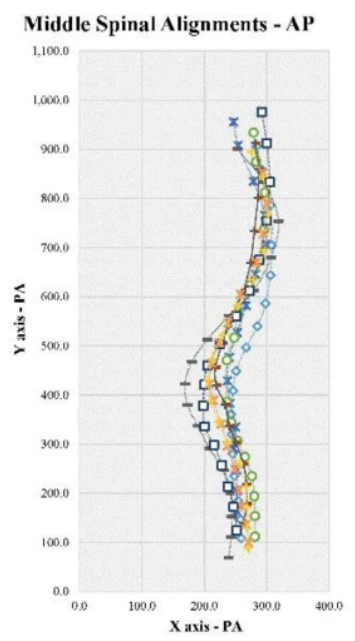

b)

Figure 2. Results: a) Scoliosis X-ray images processed with landmark detection framework with EfficientDet model 1) vertebral focused landmark points, 2) centroid points of each vertebrae L5-L1 and T1-T12, 3) spinal centerline in frontal plane, 4) intervertebral discs' points; b) frontal spinal alignment based on coordinate points for 8 samples

Figure 2 illustrates results of image processing and vertebral landmark detection for two scoliotic cases and also resulting frontal spinal alignments (MSA) for 8 typical samples. In table 1 average pixel coordinates and standard deviations of 18 scoliosis patients with predominantly thoracic and thoracolumbar AIS were calculated for each vertebral level from T1-T12 and L1-L5 respectively. These results are of crucial importance for developing a new AIS classifications, as current King and Lenke classification schemes are 2D and still rely on manual observation of X-ray radiographs.

Table 1. Average pixel coordinates and standard deviations of 18 scoliotic patients.

\begin{tabular}{|c|c|c|c|c|c|c|c|c|c|c|c|c|c|c|c|c|c|}
\hline & T1 & T2 & T3 & T4 & T5 & T6 & T7 & T8 & T9 & T10 & T11 & T12 & L1 & L2 & L3 & L4 & L5 \\
\hline $\begin{array}{l}\text { Av } \\
\text { gX }\end{array}$ & 1.8 & 2.0 & 2.2 & 2.4 & 2.5 & 2.7 & 2.9 & 3.1 & 3.4 & 3.7 & 4.1 & 4.4 & 4.8 & 5.1 & 5.5 & 5.1 & 6.0 \\
\hline $\begin{array}{l}\text { Av } \\
\text { gY }\end{array}$ & 1.7 & 2.0 & 2.1 & 2.4 & 2.6 & 2.7 & 3.0 & 3.2 & 3.5 & 3.8 & 4.2 & 4.5 & 4.9 & 5.3 & 5.6 & 5.9 & 6.2 \\
\hline $\begin{array}{c}\text { Std } \\
\mathrm{X}\end{array}$ & 82.1 & 60.3 & 40.3 & 18.2 & 32.9 & 58.1 & 81.0 & 101.4 & 121.0 & 140.8 & 155.9 & 175.1 & 201.4 & 231.9 & 267.3 & 303.7 & 331.9 \\
\hline $\begin{array}{c}\text { Std } \\
\text { Y }\end{array}$ & 75.8 & 54.3 & 34.6 & 18.0 & 32.6 & 57.4 & 79.9 & 99.8 & 118.7 & 138.1 & 152.5 & 170.6 & 195.9 & 225.6 & 260.0 & 295.0 & 322.5 \\
\hline
\end{tabular}

By knowing the coordinates of the centroids and shape of the MSA line, we are able to calculate various parameters of deformity like Cobb angles, transpositions and to 
predict the therapy outcomes after certain time of treatment [6]. This also allows us to compare 3D optical non-invasive approaches against X-ray images to confirm their reliability and their potential in predicting MSA without exposing patients to considerably high doses of radiation in diagnosis as well as in monitoring follow-up visits [7].

\section{Conclusions}

In this paper, we presented an approach which process AP X-ray radiographs of the AIS patients, recognizes vertebral anatomical landmarks, calculates vertebral and intervertebral centroids and their coordinates and takes care of inter-dependency between each vertebra by addressing low contrast images and ambiguous boundaries. Our cascaded model of object detector gives promising results on accurate landmark localization and the automatic MSA estimation in 2D. As AIS is a highly complex 3D deformity, second plane (sagittal) should be also considered. In that course we expect that the same approach can be applicable for sagittal images and then we will be able to generate 3D curve of the deformity. Research results presented in this paper can lead us towards a new 2D and 3D classification of AIS and provide us a deeper insight in the nature of the spinal curvature.

\section{Acknowledgement}

This project received funding from the European Union's H2020 research and innovation programme under the Marie Sklodowska - Curie (MSC) grant agreement No 892729.

\section{References}

[1] Yi J, Wu P, Huang Q, Qu H, Metaxas DN. Vertebra-focused landmark detection for scoliosis assessment. Proceedings of the IEEE 17th International Symposium on Biomedical Imaging (ISBI); 2020 Apr; p. 736-740.

[2] Wu H, Bailey C, Rasoulinejad P, Li S. Automatic landmark estimation for adolescent idiopathic scoliosis assessment using BoostNet. Proceedings of the International Conference on Medical Image Computing and Computer-Assisted Intervention; 2017 Sep; Springer; p. 127-135.

[3] Sekuboyina A, Bayat A, Husseini ME, Löffler M, Rempfler M, Kukačka J, Tetteh G, Valentinitsch A, Payer C, Urschler M, Chen M, et.al. VerSe: A vertebrae labelling and segmentation benchmark. 2020 Jan.

[4] Tan M, Pang R, Le QV. Efficientdet: Scalable and efficient object detection. Proceedings of the IEEE/CVF Conference on Computer Vision and Pattern Recognition; 2020 Jun; p. 10781-10790.

[5] Khanal B, Dahal L, Adhikari P, Khanal B. Automatic cobb angle detection using vertebra detector and vertebra corners regression. In International Workshop and Challenge on Computational Methods and Clinical Applications for Spine Imaging; 2019 Oct; Springer; p. 81-87.

[6] Cukovic S, Ghionea I, Lukovic V, Taylor W, Complex curvature analysis of the middle spinal line in non-ionizing 3D diagnosis of adolescent idiopathic disorders. International Conference on Biological Information and Biomedical Engineering; 2018 Jul 6-8; Shanghai, China, IEEE; 2018. p. 284-287.

[7] Cukovic S, William RT, Heidt C, Devedzic G, Lukovic V, Bassani T, Transpositions of intervertebral centroids in adolescents suffering from idiopathic scoliosis optically diagnosed. CMBBE 2019. Lecture Notes in Computational Vision and Biomechanics. 2019; 36: p. 133-141. 